\title{
Analysis and Applications of Complex Social Networks
}

\author{
Katarzyna Musial, ${ }^{1}$ Piotr Bródka, ${ }^{2}$ and Pasquale De $\mathrm{Meo}^{3}$ \\ ${ }^{1}$ University of Technology Sydney, Sydney, NSW, Australia \\ ${ }^{2}$ Wroclaw University of Science and Technology, Wroclaw, Poland \\ ${ }^{3}$ University of Messina, Messina, Italy \\ Correspondence should be addressed to Katarzyna Musial; katarzyna.musial-gabrys@uts.edu.au
}

Received 22 November 2017; Accepted 28 November 2017; Published 27 December 2017

Copyright (C) 2017 Katarzyna Musial et al. This is an open access article distributed under the Creative Commons Attribution License, which permits unrestricted use, distribution, and reproduction in any medium, provided the original work is properly cited.

Social networks are everywhere and research aiming at analysing and understanding these structures is growing year by year as its outcomes enable us to understand different social phenomena including social structures evolution, communities, spread over networks, and dynamics of changes in networks. This huge interest in the analysis of large-scale social networks resulted in a lot of new approaches, methods, and techniques but with every advancement in this area, we uncover new challenges and new levels of complexity in the network universe that are far from being explored and addressed. The increasing complexity of tasks to be performed in terms of network analysis together with the volume, variety of social data about people and their interactions, and velocity with which this data is generated in the online world pose new requirements and challenges on researchers. One of them is: how to build accurate methods that would be able to cope with this vast amount of data. This issue is a result of an attempt to address these emerging challenges.

One of the goals of this special issue is to identify the areas where social network analysis can be applied and generate knowledge not accessible through other types of analysis. We also aimed at showing that analysis of large-scale, real-world social networks underpinned by fundamental research is the direction to take when it comes to the future of complex social network analysis. We emphasize that in the world of network science fundamental research and application-driven research are equally important and they need to go together to generate significant academic, societal, and commercial impact.
The variety of papers published in this special issue shows that there is a long list of topics that have not yet been comprehensively researched. These papers also show the future challenges and trends in analysis and applications of complex social networks. The articles in this issue cover topics starting from very practical and application-based ones such as (i) case study on evolution of collaborative systems, (ii) community detection and analysis, (iii) link dynamics, (iv) spreading processes including modelling behaviour of rumour maker and influence maximization problem, and (v) frangibility in social consensus system but it also presents some research more fundamental in its nature including optimisation of structural network properties and measuring complexity of networks.

Published papers show that although all of the presented topics have been researched for many years now, there is still space and need for new contributions. Challenges change their nature as we face vast amounts of heterogeneous data that are continuously generated. Network dynamics, communities, spread analysis, consensus formation, network complexity, and structural properties are topics that are trending in research community all over the world. Those are very hard problems to address because of their complexity originating from two sources (i) system, variety of connections, attributes of nodes and connections, nontrivial structure and dynamics of a system, and (ii) process, evolution driven by variety of factors including external ones that are very hard to capture, spreading over complex structure of multiple processes, or needed process adaptation connected with evolving structure. Thus, there is a continuous need to create 
cross-disciplinary teams that would work on those challenges having a holistic view of the problem. So our work does not stop here, and we aim at continuing to bring together people from different fields to work on the topics covered within this special issue.

\section{Acknowledgments}

This special issue is an outcome of hard work of a number of people and could not happen without the support of our collaborators. This work was possible thanks to the researchers who provided their anonymous reviews. Finally, we are most grateful to the authors for their valuable contributions and for their willingness and efforts to improve their papers in accordance with the reviewers' suggestions and comments.

Katarzyna Musial Piotr Bródka

Pasquale De Meo 


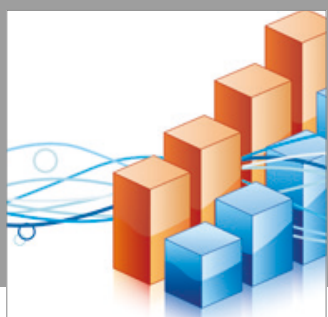

Advances in

Operations Research

vatersals

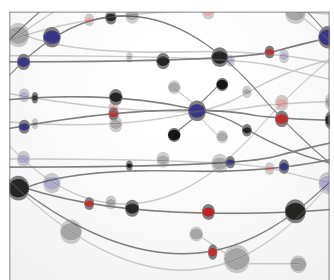

\section{The Scientific} World Journal
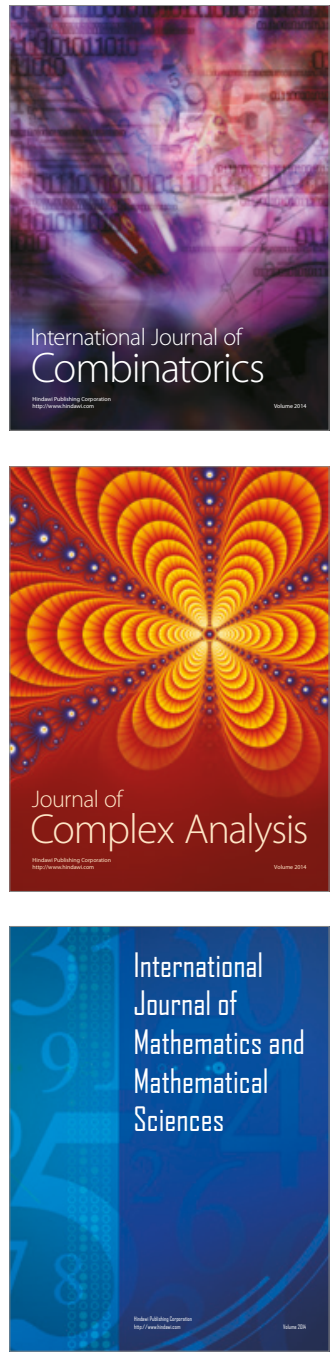
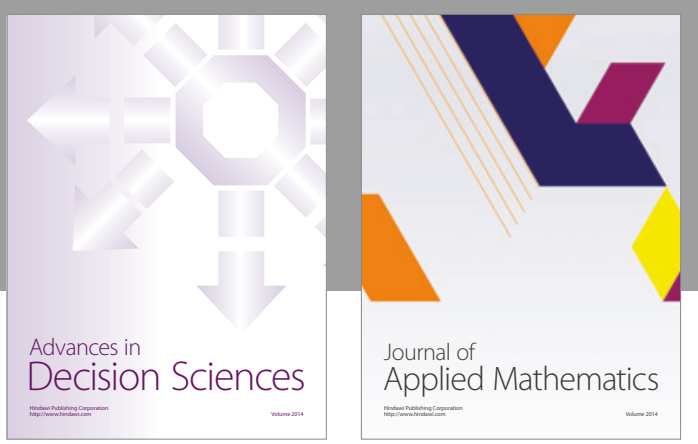

Algebra

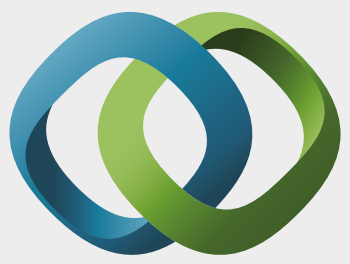

\section{Hindawi}

Submit your manuscripts at

https://www.hindawi.com
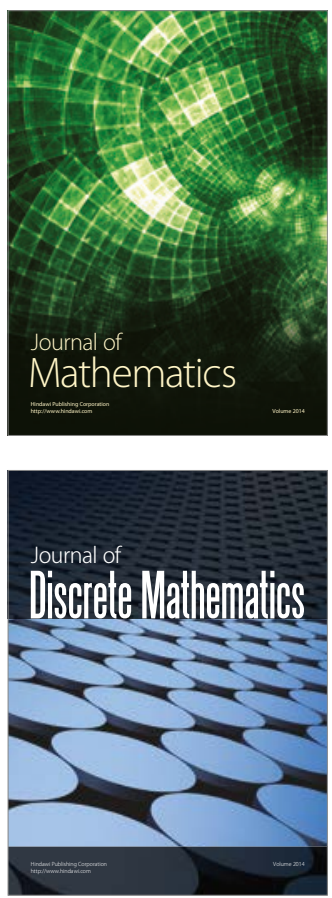

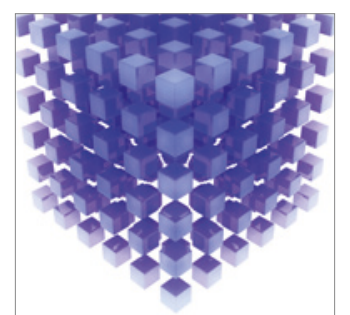

Mathematical Problems in Engineering
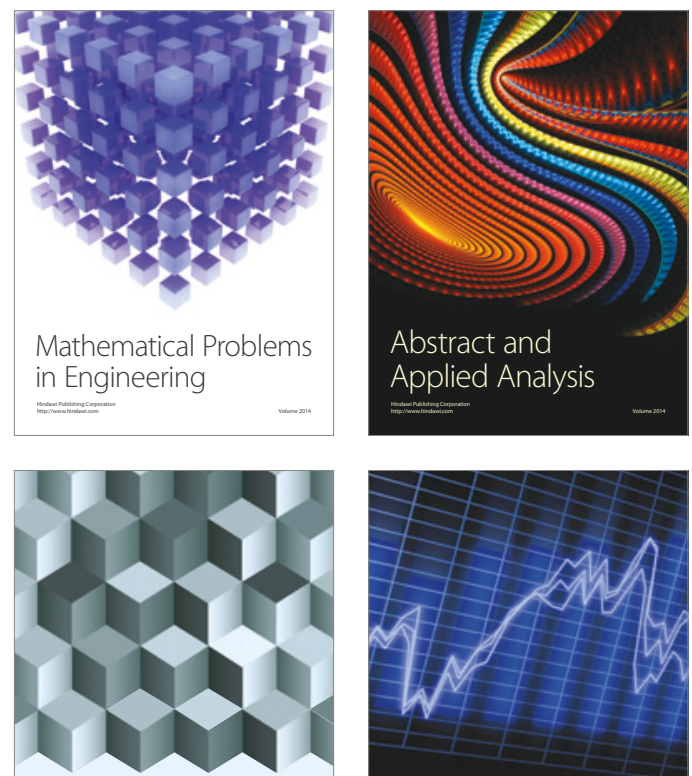

Journal of

Function Spaces

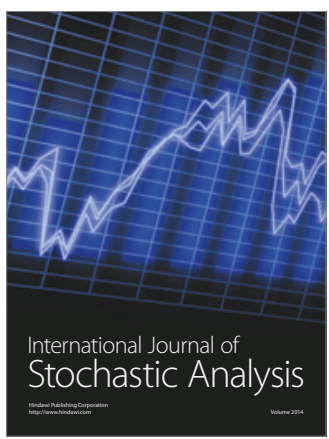

Probability and Statistics
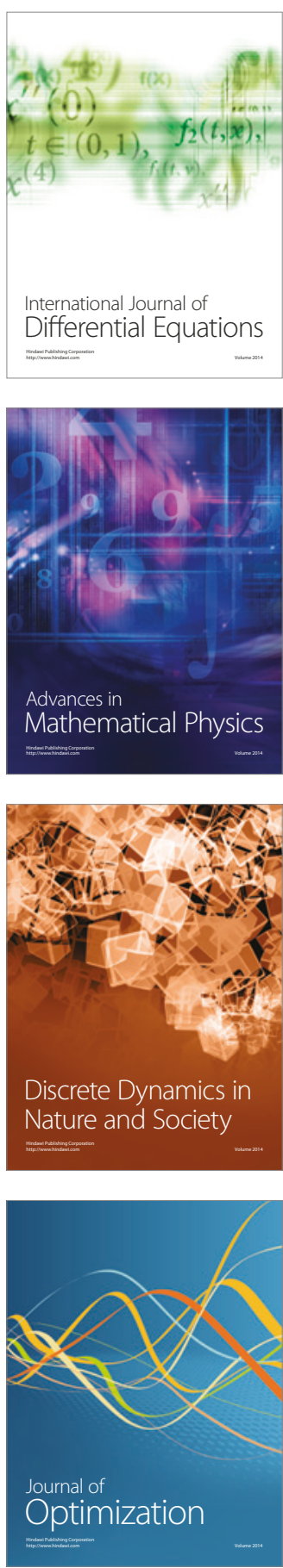